$\begin{array}{ll}\text { Italique } & \text { Italique } \\ \text { Poésie italienne de la Renaissance }\end{array}$

XIV | 2011

Varia

\title{
Un' altra implicazione senese per un sonetto di Galeazzo di Tarsia
}

Luca Milite

\section{Q OpenEdition}

1 Journals

\section{Edizione digitale}

URL: http://journals.openedition.org/italique/335

DOI: 10.4000/italique.335

ISSN: 1663-4438

\section{Editore}

Librairie Droz

\section{Edizione cartacea}

Data di pubblicazione: 1 gennaio 2011

Paginazione: 133-143

ISBN: 978-2-600-01536-3

ISSN: 1423-3983

\section{Notizia bibliografica digitale}

Luca Milite, «Un' altra implicazione senese per un sonetto di Galeazzo di Tarsia », Italique [Online], XIV | 2011, online dal 25 juin 2014, consultato il 02 mai 2019. URL : http://journals.openedition.org/ italique/335; DOI : 10.4000/italique.335

(C) Tous droits réservés 
LuCA Milite

U N'A L T R A I M P I C A Z I ONE S E N E S E PER UN SONETTO D I

G A LE A Z Z O D T A R I A 



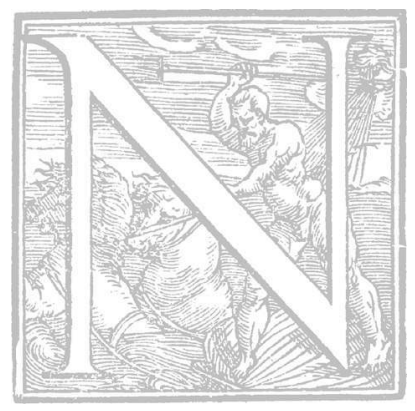

el 1998 Massimo Danzi $i^{\mathrm{I}}$ diede notizia di una redazione fino ad allora sconosciuta del sonetto tarsiano Come in limpido vetro $\mathrm{o}$ in onda pura, numero 16 nell'edizione Bozzetti, rinvenuta adespota nel manoscritto I.XI.49 della Biblioteca Comunale degli Intronati di Siena entro un gruppo di rime attribuite esplicitamente all'accademico Deserto Antonio Barozzi. Del medesimo Barozzi nella medesima carta (c.27r) del medesimo manoscritto si trova anche un sonetto che il Danzi riproduceva e giudicava, mi pare a ragione, imitazione di quello di Galeazzo, ${ }^{3}$ Come puro cristallo in cui del sole, e in un'altra carta (c. $38 r$ ), aggiunta al codice e di altra mano, due lunghi frammenti in versi sciolti. Il primo di questi, di 23 versi endecasillabi (ma l'undicesimo è un novenario) contiene «gli elementi, nonché le principali tounures sintattiche del sonetto di Galeazzo», ${ }^{4}$ mentre il secondo, composto di 36 versi di cui $i$ 19, 24 e 25 endecasillabi e gli altri settenari, si riferisce invece al sonetto del Barozzi.

Purtroppo che cosa fossero precisamente $i$ due frammenti e a chi fossero da attribuire non era possibile dire allora e non è possibile nemmeno oggi. Il Danzi formulava con acume e perizia due diverse ipotesi, entrambe sensate e però entrambe di necessità insoddisfacenti, che spero di riassumere qui con bastante precisione.

I) Il primo frammento è un primo abbozzo informe del sonetto di Galeazzo; assai probabilmente non un autografo, ma la copia di uno o più autografi. Va da sé che anche il secondo frammento sia un abbozzo, ma del Barozzi.

2) I due frammenti sono rifacimenti in sciolti dei due sonetti, di Galeazzo e del Barozzi, entrambi attuati di getto dal Barozzi.

Succede spesso che una nuova scoperta ponga piu problemi di quanti ne risolva; allo studioso non rimane che schedare coscienziosamente gli elementi e le implicazioni possibili e formulare ipotesi attendibili in attesa che le cose si possano chiarire di più in futuro con nuove scoperte. Questo è stato fatto dal Danzi e questo spero di riuscire a fare io a mia volta in questa circostanza.

Non mi allontano, per ora, dallo studio del Danzi e riproduco qui di seguito il sonetto di Galeazzo secondo l'edizione Bozzetti, ma 
con l'aggiunta in apparato delle varianti del codice senese, sotto la sigla S:

Come in limpido vetro o in onda pura, Se il destr'occhio del ciel risplende in lui, Mirar si può quel che ne' raggi sui

Debil vista mirar non s'assicura,

Così la mia, ch'altro veder non cura,

Perde, Donna real, mirando in vui

Che siete un nuovo sole oggi fra nui:

Ch'occhio non sano a gran splendor non dura.

Ma se mi volgo al cor che d'ogni parte

Riceve il folgorar del vago viso,

Non splende raggio in lui ch'ei non mi mostri.

Dunque a che tormi il sol de gli occhi vostri

Se il veggio assai via men se in lui m'affiso

E lo scopro in me stesso a parte a parte?

2 risplende] riflette $\mathrm{S} 4 \mathrm{mirar}$ a mirar $\mathrm{C}, \mathrm{V} \quad 6$ Donna real] (o lume gentil) $\mathrm{S} 7$ siete...oggi] quasi un nuovo sol sete $\mathrm{S}$ 8 splendor] fulgor $\mathrm{C} 9$ se] s'io $\mathrm{S}$ io il...viso] la di voi luce in se stesso $\mathrm{S}$ folgorar] balenar $\mathrm{C}$ vago] vostro $\mathrm{C}, \mathrm{V}$, $\mathrm{NR}$ I I splende...mi] è sì chiaro in lei ch'ei non si $\mathrm{S}$ I I lui] voi $C, V, N R$ ch'ei] che $C$ I 3 Se il] S'io lo $S$ assai via men se in lui m'affiro] meno assai qualor l'affiro $C$ assai men s'a lui m'appresso S I4 in me stesso] da lunge $S$

2 Se il] Se 'l C, V destr'] destro C, V, S 3 ne'] ne i NR S 4 assicura] assecura NR asicura $S 7$ siete] sete C, V, NR I I nuovo] novo $\mathrm{C}, \mathrm{V}$ I 2 de gli] degli NS I 3 Se il] Se '1 $\mathrm{V}$ se in] s'in V I4 scopro] scuopro $\mathrm{C}, \mathrm{V}$

$E$ ora il frammento in sciolti, che Danzi sigla $A$. Lo riproduco con l'aggiunta della punteggiatura, correggendo al $v .7$ la (lezione del manoscritto) in lo ed accogliendo al v. 20 l'emendamento del Danzi, bel anzi che nel:

Come in limpido vetro o in onda chiara,

Se 'l bel lume del sol riflette in lui,

$\mathrm{Si}$ scorge in parte quel vago splendore

Ch'in se stesso mirar alcun non puote, 
Così quel bel ch'in voi natura pose

Vo rimirando nel mio cor sovente,

Ch'ivi Amor lo scolpì con la sua mano,

E riceve da voi ogni suo lume;

Ma s'io riguardo in voi, tanta è la luce

De' sereni occhi vostri, che mirarvi

Io pur non posso e questo è certo,

Ch'occhio non san a gran splendor non dura.

Quando ti son appresso ogni mio senso

Intento è a procurar se in te propria

Potesse mai conoscere quel bello

Che v'ascose natura, onde tu fusse

Esempio al'altre, oltre a le belle bella;

Onde nel cuor io non ti miro al'hora,

Né l'occhio può mirarti e resto privo

Di così bel vedere. Onde, se noia

T'è ch'io ti veggia, stammi ogni hora a presso,

Ch'el mio senso visivo non vedratti,

E men nel cuor potrò mirarti mai.

Già il Danzi aveva sospettato il carattere non unitario del frammento, in base alla ripetizione del concetto della natura ( $\mathrm{vv}$. 5 e I 5 ) e soprattutto dal cambio del pronome con cui ci si rivolge alla donna, prima il voi, e poi il tu. Secondo me si tratta di due componimenti diversi: il primo, caratterizzato dall'uso del voi, comprende $i v v$. I-I2 e finisce con il verso sentenzioso e lapidario Ch'occhio non san a gran splendor non dura, che sembra tolto di peso dal sonetto di Galeazzo per servire da explicit. Mi pare molto più probabile, infatti, che gli sciolti siano, piuttosto che il primo abbozzo d'autore di Come in limpido specchio od onda pura, un tentativo di rifacimento, ed anche piuttosto maldestro. La prima parte del frammento sarebbe dunque la rielaborazione dei primi tre quarti del sonetto tarsiano, che contengono la parte teorica che serve utilitaristicamente alla dimostrazione dell'assunto finale: è perfettamente inutile che la donna si sottragga alla vista del suo amante, perché questi la contempla meglio da lontano. Insomma il Tarsia utilizza con arguzia cortigiana la descrizione di un fenomeno naturale per dimostrare come la donna debba farsi vedere da lui, essendo assolutamente futile, date le premesse e alla luce delle più avanzate conoscenze, ogni possibile tentativo di contraddire al suo desiderio.' 
L'ultima parte, la conclusione del ragionamento del sonetto, è imitata nel secondo componimento in sciolti, insieme individuabile nella sua alterità ma dipendente, quanto alle premesse, dal primo, come in un dittico di sonetti o in due strofe di rispetti continuati; si spiegherebbe in questa prospettiva la ripetizione del concetto della natura, insieme richiamo e riepilogo tematico, anche se bisogna dire che stride parecchio il passaggio dal voi al tu; ma consideriamo che stiamo parlando forse di un'opera di un dilettante e quasi certamente neanche compiuta. L'imitazione dell'ultima terzina è tra l'altro attuata per caricatura, esagerando quanto di intellettualistico vi era nell'originale e nel contempo, forse per desiderio di amplificazione, forse per tema di non esser stati abbastanza chiari, esplicitando ogni possibile allusione con un ordine diretto in un linguaggio veramente troppo colloquiale e prosastico anche per un qualsiasi strambottista dell' età precedente: 'dato che la tua vicinanza mi abbaglia si che non posso guardarti, e d'altra parte avendo te vicino non posso fare altro che cercare di guardare te direttamente (questo significherebbe propria al v. I4) invece che nel mio cuore, allora, se ti dà fastidio che io veda la tua bellezza, è proprio vicino a me che devi stare'.

Il diagramma procede dunque secondo me dal semplice al complesso; da un'opera compiuta (il sonetto di Galeazzo) si passa ad un esercizio di rielaborazione per accumulo e superfetazione (gli sciolti) e non viceversa. Resta da vedere se il sonetto di Galeazzo sia stata l'unica fonte degli sciolti, o se qualcosa della sovrabbondante concettosità di $A$ sia non solo farina del sacco del rifacitore, ma provenga da una fonte cui attinse anche Galeazzo; è questo, secondo me, il caso.

Come aveva già fatto notare il Danzi, costituiva una certa novità questo documento della fortuna senese di Galeazzo: a Siena, in ambienti che devono riferirsi alla locale (ma con importanti implicazioni napoletane) ${ }^{6}$ Accademia degli Intronati, circolava un sonetto tarsiano in una primitiva redazione; e aveva fatto scuola, si può dire, perché era stato imitato prima dal Barozzi Deserto Intronato, e poi, assieme al Barozzi, da un anonimo (ma probabilmente anch'egli Intronato) le cui carte finirono nel nostro manoscritto degli Intronati di Siena. Ma anche la nascita, la prima ispirazione di Come in limpido vetro o in onda pura ha precise connotazioni senesi, almeno questa è la mia opinione; il che spiegherebbe meglio anche la curiosa fortuna senese del sonetto del Tarsia. 
Credo di aver identificato il testo ispiratore sia di S (cioè del sonetto del Tarsia nella redazione del manoscritto senese) che di $A$ (il frammento in sciolti). É la prima parte della dedicatoria di Alessandro Piccolomini a Laodomia Forteguerri nell'Institutione di tutta la vita de l'homo,' Venezia, Gerolamo Scotto, I542. La riproduco qui di seguito, con una nuova punteggiatura, annotando in calce le somiglianze con $S$ e $\operatorname{con} A$ :

Mi stava quest'autunno passato, un dì fra gli altri, sì com'ero solito su 'l mezo giorno di fare, nel giardin mio, sott'una verdura intessuta d'edera, in me medesmo raccolto, virtuosissima Madonna Laudomia; e havendo poco innanzi letto il XXXI Canto del Paradiso di Dante, dove de la somma felicità si ragiona, il qual voi già, con gran mio stupore, se ben vi ricordate, m'interpretaste, tutto m'ero col pensiero profondamente rivolto a molte bellissime cose che voi sopra la felicità umana e angelica dottissimamente mi ragionaste. E, una cosa da l'altra sovvenendomi, cominciai con molta più maraviglia, considerando sì belle cose, a stupir del giuditio vostro, che io non feci in quel giorno che, raccontandole voi, le raccolsi. Il qual miracolo d'altronde nascer non può, se non che a la presentia vostra il vostro bello ${ }^{a}$ m'abbagliava così la vista del senso e de l'intelletto ${ }^{\mathrm{b}}$ che 'l saper vostro e 'l valor de le parole vostre non discernevo. Onde, sì come accascar suole a coloro che, volendo nel corpo solare conoscere alcune cose, forza è che, per meglio vederle, non in esso, ma in qualche corpo limpido, ${ }^{\mathrm{c}}$ donde egli co' i suoi razi refletta, ${ }^{\mathrm{c}}$ fissamente riguardino, così a me parimente adiviene: che per voler distintamente cognoscer la virtù vostra mi fa di mestieri che non in voi stessa, ${ }^{\mathrm{d}}$ che da lungi il mio giuditio vincete, ${ }^{\mathrm{e}} \mathrm{ma}$ in qualche luogho dove la vostra vera imago risieda rivolga gli occhi del mio pensiero. Né luogho alcuno credo io che si truovi donde i raggi del valor vostro con più forte imago reflettino ${ }^{c}$ che da 'l mio core, il qual da ogni parte ${ }^{\mathrm{f}}$ mi mostra voi. ${ }^{\mathrm{g}}$ Onde nasce che molto più (com'ho detto) mi riempìan di stupore, send'io lontano, ${ }^{\mathrm{h}}$ quelli ingeniosissimi vostri ragionamenti che sopra al detto Canto di Dante intorno a l'humana e angelica felicità mentre che gli facevate mi s'impresser ne l'anima, che send'io presente ${ }^{\mathrm{i}}$ non m'aveniva $[\ldots]$

a. quel bel ch'in voi natura pose A 5; quel bello / Che v'ascose natura A i 5-I6.

b. Quando ti son appresso ogni mio senso / Intento è a procurar A I 3-4; Ch'el mio senso visivo non vedratti A 22; Ch'occhio non sano a gran splendor non dura S 8; Ch'occhio non san a gran splendor non dura A i 2.

c. Come in limpido vetro o in onda pura, /Se il destr'occhio del ciel riflette in lui, 
/ Mirar si può S I-3; Come in limpido vetro o in onda chiara, / Se 'l bel lume del sol riflette in lui, / Si scorge in parte quel vago splendore / Ch'in se stesso mirar alcun non puote $\mathrm{A} I-4$.

d. in se stesso A 4 ; in te propria $\mathrm{A}_{\mathrm{I}}$; ; in se stesso $\mathrm{S}_{\mathrm{I}}$.

e. Perde, o lume gentil, mirando in vui S 6

f. al cor che d'ogni parte $S_{9}$.

g. Non è sì chiaro in lei ch'ei non si mostri S I I

h. e lo scopro da lunge S i 4 .

i. s'a lui m'appresso S I 3; Quando ti sono appresso A I 3; stammi ogni hora a presso A $2 \mathrm{I}$.

Il Piccolomini prende spunto dal ricordo di dotte conversazioni sul Paradiso di Dante per lodare la dedicataria della sua opera; il suo discorso rivela insieme la sua cultura scientifica e la sua passione per la Commedia; il suo linguaggio, in buona prosa cinquecentesca e con galanteria rinascimentale, riprende il lessico scientifico volgare caratteristico del poema dantesco. Alludo in particolare all'uso del verbo reflettere, che non si riscontra in Petrarca ed è comune alla prefatoria, ad $A$ e S, e che più tardi verrà sostituito dal Tarsia col petrarchesco risplendere.

Reflettere è proprio dei paragoni scientifici della Commedia:

Purg. 25, 91-93: E come l'aere, quand'è ben piorno, / Per l'altrui raggio che 'n sé si reflette, / Di diversi color diventa adorno;

Par. 30, 106-108: Fassi di raggio tutta sua parvenza / Reflesso al sommo del mobile primo, / Che prende quindi vivere e potenza;

Par. 33, i 8-20: E l'un dall'altro come iri da iri / Parea reflesso, e l'altro parea foco / Che quinci e quindi igualmente si spiri;

Par. 33, I 27-31: Quella circulazion che sì concetta / Pareva in te come lume reflesso, / Dalli occhi miei alquanto circunspetta, / Dentro da sé, del suo colore stesso, / Mi parve pinta della nostra effige.

Petrarca, in analogo contesto, preferirà invece risplendere:

Rvf 95.9-10: Poi che vostro vedere in me risplende, / Come raggio di sol traluce in vetro.

$E$ in $S$ al v. 2 riflette e non risplende si legge, avendo Galeazzo ereditato, nella prima redazione, il lessico della sua fonte (solo più tardi il Tarsia sostituirà riflette con risplende). S insomma è il punto di partenza che conferma il percorso variantistico dell'autore come lo des- 
crisse già Bozzetti, «dominato essenzialmente dalla ricerca di un lessico sempre più petrarchesco e insieme da una progressiva attenuazione delle forme troppo colloquialis;; un che di colloquiale, anzi, potremmo dire, prosastico, rimane sempre anche nell'ultima redazione, ed è dovuto al peccato originale di questo sonetto, il suo essere in massima parte derivato da una prosa. Ė la conseguenza del fatto generale che il sonetto è naturalmente un genere metrico anche interlocutorio, epistolare, e quindi sono possibili interferenze, come si vede nelle lettere e nelle rime del Bembo; né sembri strano che lo scambio avvenga anche tra autori diversi, perché quello del Tarsia e del Piccolomini non è un caso isolato: posso citare anche il sonetto LXI delle Rime del Rota, la cui prima quartina,

Dentro il thesor de la memoria mia Vi serbo sempre, e ben posso thesoro Chiamarlo, se i robin, le perle e l'oro Dentro vi son che vi fan bella e ria?

deriva da un passo di una lettera di Jacopo Bonfadio a Volpino Olivi, del I54I e a stampa nel I543:

Questo ha fatto sì ch'io vi ho avuto sempre in memoria, o per dir meglio, ne i tesori della memoria, ché così ben li posso chiamare, poiché ci siete entro voi, che per dire il vero voi possedete mille ricchezze d'animo e d'ingegno. ${ }^{\circ}$

Torno alla lettera dedicatoria e ai suoi rapporti col frammento $A$ e la redazione $S$, poiché è tempo di argomentare meglio la tesi che fino ad ora avevo data per scontata, che la prosa è alla base sia del sonetto di Galeazzo che del frammento $A$, che però è effettivamente in gran parte un rifacimento di $S$.

Il fatto è che l'elemento che ho segnalato con la nota a), quel bel / quel bello / il vostro bello, accomuna la prosa ad A mentre non è presente in $S$; se ne dedurrebbe quindi che $A$, rifacimento di $S$ (le coincidenze tra $i$ due testi sono ineludibili) abbia risentito, per memoria o volontaria consultazione, della lettera dedicatoria del Piccolomini.

Per accettare meglio questa tesi, ovviamente, bisogna escluderne un'altra, che di primo acchito sembra forse più semplice e naturale: che $A$ sia il primo abbozzo di $S$, che per questo contiene un elemento presente nella prosa che poi è stato abbandonato nella redazione in 
sonetto, dato che inoltre sembra intuitivamente accettabile e lineare l'ipotesi di un'evoluzione in tre stadi: dalla prosa alla rima attraverso la fase intermedia del verso sciolto.

Si ricorderà che il manoscritto di Siena contiene, sulla stessa carta aggiunta, della stessa mano diversa dalle altre, oltre ad $A$, un frammento in settenari sciolti il cui contenuto è chiaramente riferibile al sonetto Come puro cristallo in cui del sole del Barozzi; ora, credo che $i$ due (o tre, se il primo a sua volta consta di due) componimenti in sciolti debbano avere la stessa natura e la stessa origine, altrimenti non si spiegherebbe che ci facciano lì insieme; e se $A$ è abbozzo di S allora è di Galeazzo, ed anche l'altro frammento deve essere suo; ma l'altro frammento non può essere suo. Se $A$ è del Barozzi, allora tutti e due gli sciolti sono rifacimenti, opera dello stesso autore, di due sonetti di autori diversi. Ma non vedo perché il Barozzi, autore di un'imitazione originale e ben fatta, abbia sentito il bisogno di rifare in sciolti ma in maniera pedissequa sia il sonetto del Tarsia che il proprio che del Tarsia era già imitazione.

Sembra inoltre difficile che un abbozro, nell'ipotesi che $A$ lo sia, contenga in nuce già perfetti o quasi gli snodi sintattici ed il lessico di un sonetto senza averne però neanche una rima: un poeta del Cinquecento è prima di tutto un rimatore, e le rime e lo schema metrico dourebbero essere parte essenziale della creazione poetica anche allo stato embrionale. Questo dourebbe valere non solo per Galeazzo, per la verità, ma anche per il Barozzi.

La mia opinione è, come avevo anticipato, che un anonimo poeta non particolarmente amico delle Muse abbia rielaborato in sciolti $i$ due sonetti del Tarsia e del Barozzi, e che questo anonimo non sia da identificare in nessuno dei due. Il Sonetto del Tarsia, giunto a Siena non si può sapere come ma sicuramente attraverso le relazioni che gli Intronati avevano con Napoli, avrà suscitato un certo interesse per l'omaggio che il Tarsia aveva tributato con la sua imitazione all'illustre filosofo senese (e Stordito Intronato) Alessandro Piccolomini; l'interesse sarebbe poi sfociato nell'imitazione da parte del Barozzi con il sonetto Come puro cristallo in cui del sole, e poi entrambi $i$ sonetti, il napoletano e il senese, sarebbero stati imitati dall'anonimo degli sciolti che avrebbe tenuto presente anche la prosa del Piccolomini che sapeva essere all'origine di tutto.

Luca Milite 
I. Massimo Danzi, Storia e fortuna senesi di un sonetto di Galeazzo di Tarsia, «Italique» I (1998), pp. 6r-78.

2. Galeazzo di Tarsia, Rime, ed. critica a c. di Cesare Bozzetti, Milano, Fondazione Arnoldo e Alberto Mondadori, i 980.

3. In una gara di erudizione scientifica, dato che il puro cristallo del Barozzi non è, come in Galeazzo, uno schermo per l'osservazione del sole, ma la sfera di cristallo che concentra i raggi incendiando gli oggetti; altra curiosità scientifica classico-rinascimentale, che aveva dato materia per un'impresa di Clemente settimo col motto candor illaesus (Giovio, Dialogo dell'imprese militari e amorose, a c. di M.L. Doglio, Roma, Bulzoni, 1978 pp. 66-7; G. Ruscelli, Le imprese illustri, Venezia, Rampazzetto, I 566, pp. I6 1-4; Plin. Nat. 37.28-29, Isid. 1 3.50).

4. Massimo Danzi, Storia e fortuna senesi di un sonetto di Galeazzo di Tarsia, cit. p. 69.

5. Analogo procedimento, ma più versato nell'ambito logico-filosofico, utilizza il Rota nel più o meno coevo sonetto Se de l'occhio del ciel l'alma gran luce (BERNARDINo RotA, Rime, a c. di L. Milite, Milano, Guanda, 2000, LXV) ove tra l'altro è apprezzabile la coincidenza della metafora classica del sole come occhio del cielo.

6. Si veda a questo proposito anche T.R. Toscano, Schede sul noviziato poetico napoletano di Vittoria Colonna, in Letterati corti accademie. La letteratura a Napoli nella prima metà del Cinquecento, Napoli, Loffredo, 2000, pp. i 8-i 9.

7. De la institutione di tutta la vita de l'homo nato nobile e in citta libera. Libri $X$ in lingua toscana. Dove e Peripateticamente e Platonicamente, intorno a le cose de l'Ethica, Iconomica, e parte de la Politica, è raccolta la somma di quanto principalmente può concorrere a la perfetta e felice vita di quello. Composti dal. S. Alessandro Piccolomini, a benefitio del Nobilissimo Fanciullino Alessandro Colombini, pochi giorni innanzi nato; figlio de la Immortale Madonna Laudomia Forteguerri. Al quale (havendolo egli sostenuto a battesmo), secondo l'usanza de $i$ compari, de i detti libri fa dono. Precede la dedicatoria di Ottaviano Scotto al Marchese e alla Marchesa del Vasto, datata I 542. La dedicatoria dell'autore alla Forteguerri è datata invece di Padova, il primo giorno de l'anno, I 540.

8. Galeazzo di Tarsia, Rime, cit. p. 53 . Bozzetti, è forse il caso di ricordare, non conosceva ancora $\mathrm{S}$.

9. B. Rota, Rime, cit.

io. Jacopo Bonfadio, Le lettere e una scrittura burlesca, testo con introduzione e commento a c. di A. Greco, Roma, Bonacci I978, I9.2. 